\title{
New vision on the new era of genome study
}

\author{
Parwinder Kaur ${ }^{1} \cdot$ Baohong Zhang ${ }^{2}$
}

Published online: 17 January 2022

(c) The Author(s), under exclusive licence to Springer-Verlag GmbH Germany, part of Springer Nature 2022

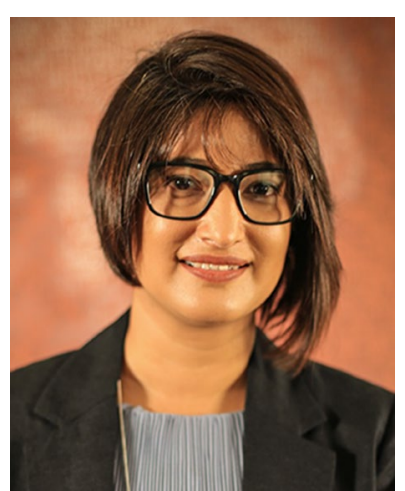

Parwinder Kaur

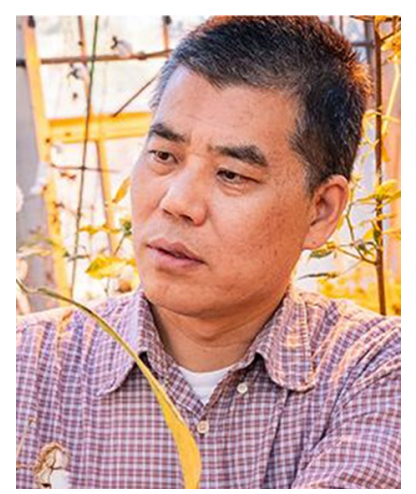

Baohong Zhang

Parwinder Kaur

parwinder.kaur@uwa.edu.au

$\triangle$ Baohong Zhang

zhangb@ecu.edu

1 UWA School of Agriculture and Environment, The University of Western Australia, Perth, WA 6009, Australia

2 Department of Biology, East Caroline University, Greenville, NC 27858, USA
Tremendous advances have been made in the field of genomics, including next generation DNA sequencing (Goodwin et al. 2016), transgenics, and current genome editing ( $\mathrm{Li}$ et al. 2021); however, this is just the beginning. Next generation genome sequencing has provided us with an exceptionally powerful tool which enables greater understanding of all aspects of planet and human health at the genome levels, including the recently rapid characterisation of the SARS-CoV-2 during the current pandemic, thus kickstarting many highly successful vaccine development programmes (Shaukat et al. 2021). In the last decade, genome-related research has been significantly expanded at an unprecedented speed, from a single gene functional study to multiple genome comparison among different genotypes in a same species and cross different species (pangenomics and metagenomics) as well as multiple omics studies (including genomics, transcriptomics, proteomics, metabolomics, and phenomics) and from structure genomics to applied genomics in which genomics has been widely used to different purposes on agriculture, environment, industry, and biomedicine. To dedicate to this field, about two decades ago, in 2000, Functional \& Integrative Genomics was born for providing a platform for both academic and industrial communities to exchange their advanced discovery. During this period, Functional \& Integrative Genomics has been becoming a major journal in the genome research field with enormous efforts of several generations of dedicated editorin-chiefs and the entire editorial boards.

Genome is a complicated set of molecules; previous studies are more focused on the coding region. However, the majority of genome region is non-coding sequences, usually called dark matter, dark sequence, or junk sequence of the genome (Sedlazeck et al. 2018). Recently, studies showed that these dark secret regions play more and more roles in all organism biological and metabolic processes, such as development process and different diseases, mainly through gene regulation, stress memory, transgenerational impact, and many others (Adelman and Egan 2017). These dark sequences include both small and long non-coding RNAs, such as microRNAs, which play versatile role in all 
organisms, including animals, plants, and microbes. Functional \& Integrative Genomics will not only report the scientific findings on coding regions, but we will also focus more on these dark secret sequences, including their identification, genome organization, biodiversity, and evolution in addition to their functional analysis and biological applications.

This new vision describes the most compelling research priorities and opportunities in genomics for the coming decade, signaling a new era in genomics for Functional \& Integrative Genomics. To face the quick development and also challenges in this field, Functional \& Integrative Genomics will continuously serve the genome research and education community by providing a reliable platform for advancing and exchanging the scientific research in all fields of genome from a single gene functional study to metagenome and multi-omics studies and from structure genome to advanced genome editing. More importantly, Functional \& Integrative Genomics will promote the application of genome research in all potential fields, including biomedicine, agricultural, environment, and industry. In this journal, we will not only publish the traditional research papers and review papers, but we also welcome publish short communications which will quickly report your significantly advanced discovery without a full story. We understand computational tools and resources and advanced experimental tools, such as CRISPR/Cas, play more and more important role in scientific discovery; thus, since 2022, we will design a specific section to publish computational and experimental tools and methods/protocols, new and revised database, and data notes for public usage. To enhance the scientific idea exchange, we will also consider several short articles, including opinion and perspective papers as well as research spotlights, particularly on hot topics with huge potentials for both fundamental research and biotechnological applications.

We assure that all manuscripts will be handled in confidence with high standard, fair, and rigorous peer review in a timely manner. We promise that you will receive the first editorial decision in less than 30 days and your manuscripts will be published in 10 days after it will be accepted. Additionally, during the initial submission process, there are no particular manuscript format requirements, which will save a lot of formatting time and allow you to quickly submit your manuscripts to Functional \& Integrative Genomics, especially when your manuscripts are transferred from other top journals. With the strong support from the scientific research community, outstanding authors, and reviewers as well as the entire dedicated editorial board, we are very optimistic that Functional \& Integrative Genomics will be moved to the next level in both journal reputation, impact of the papers published, and the audience.

January 2022.

\section{References}

Adelman K, Egan E (2017) NON-CODING RNA more uses for genomic junk. Nature 543:183-185

Goodwin S, McPherson JD, McCombie WR (2016) Coming of age: ten years of next-generation sequencing technologies. Nat Rev Genet 17:333-351

Li C, Brant E, Budak H, Zhang B (2021) CRISPR/Cas: a Nobel prize award-winning precise genome editing technology for gene therapy and crop improvement. J Zhejiang Univ Sci B 22:253-284

Sedlazeck FJ, Lee H, Darby CA, Schatz MC (2018) Piercing the dark matter: bioinformatics of long-range sequencing and mapping. Nat Rev Genet 19:329-346

Shaukat A, Hussain K, Shehzadi N (2021) COVID-19 vaccines: development, strategies, types and vaccine usage hesitancy. VacciMonitor 30:145-152

Publisher's note Springer Nature remains neutral with regard to jurisdictional claims in published maps and institutional affiliations. 